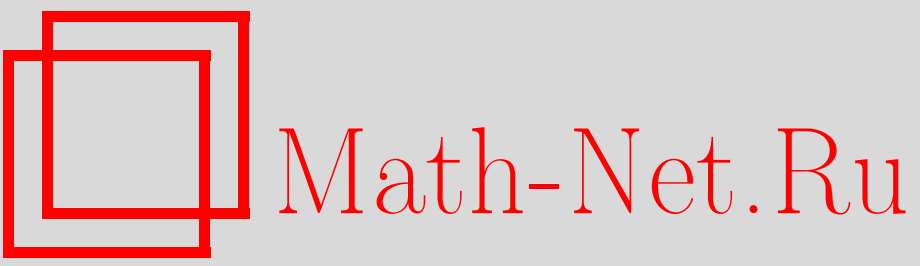

Ю. Е. Безмельницына, С. В. Корнев, В. В. Обуховский, Негладкие интегральные направляющие потенциалы в задаче об асимптотическом поведении траекторий некоторых классов функционально-дифференциальных включений, Итоги науки и техн. Сер. Соврем. мат. и ее прил. Темат. обз., 2020, том 186, 13-20

DOI: https://doi.org/10.36535/0233-6723-2020-186-13-20

Использование Общероссийского математического портала Math-Net.Ru подразумевает, что вы прочитали и согласны с пользовательским соглашением

http: //www. mathnet.ru/rus/agreement

Параметры загрузки:

IP : 54.197 .217 .227

26 апреля 2023 г., 12:34:43 


\title{
НЕГЛАДКИЕ ИНТЕГРАЛЬНЫЕ НАПРАВЛЯЮЩИЕ ПОТЕНЦИАЛЫ В ЗАДАЧЕ ОБ АСИМПТОТИЧЕСКОМ ПОВЕДЕНИИ ТРАЕКТОРИЙ НЕКОТОРЫХ КЛАССОВ ФУНКЦИОНАЛЬНО-ДИФФЕРЕНЦИАЛЬНЫХ ВКЛЮЧЕНИЙ
}

\author{
(c) 2020 г. Ю. Е. БЕЗМЕЛЬНИЦЫНА, С. В. КОРНЕВ, В. В. ОБУХОВСКИЙ
}

\begin{abstract}
АннотАция. В настоящей работе представлен новый метод решения задачи об асимптотическом поведении траекторий управляемых систем, описываемых функционально-дифференциальными включениями с невыпуклозначными правыми частями. Основным инструментом решения данной задачи является метод негладких интегральных направляющих потенциалов.
\end{abstract}

Ключевые слова: асимптотическое поведение, функционально-дифференциальное включение, нормальное мультиотображение, негладкий интегральный направляющий потенциал.

\section{NONSMOOTH INTEGRAL DIRECTION POTENTIALS IN THE PROBLEM OF THE ASYMPTOTIC BEHAVIOR OF TRAJECTORIES OF SOME CLASSES OF FUNCTIONAL DIFFERENTIAL INCLUSIONS}

\author{
(c) 2020 YU. E. BEZMELNITSYNA, S. V. KORNEV, V. V. OBUKHOVSKII
}

\begin{abstract}
In this paper, we present new methods for solving the problem of the asymptotic behavior of trajectories of control systems governed by functional differential inclusions with nonconvex-valued right-hand sides. The main tool for solving this problem is the method of nonsmooth integral directing potentials.
\end{abstract}

Keywords and phrases: asymptotic behavior, functional differential inclusion, normal multimap, nonsmooth integral directing potential.

AMS Subject Classification: 34K09, 34C37, 34A60, 34D05, 34E10, 34H15

1. Введение. В последние годы сфера применения метода направляющих функций, основы которого заложили М. А. Красносельский и А. И. Перов (см., например, [5,6], а также $[1,13,24]$ и ссылки в них), была расширена на изучение качественного поведения решений дифференциальных уравнений и включений различных типов, включающее, в частности, изучение бифуркационного явления (см., например, $[3,6,15,19,21-23])$, а также асимптотического поведения решений (см., например, $[2,4,10,16-18,22])$.

В представленной работе мы определяем понятие интегрального направляющего потенциала для функционально-дифференциальных включений и применяем его для изучения асимптотического поведения решений включений как с выпуклозначной, так и с невыпуклозначной правой

Работа выполнена при поддержке Министерства образования и науки РФ (проект № 1.3464.2017/4.6) и Российского фонда фундаментальных исследований (проект № 17-51-52022). 
частью. Такой тип поведения решений тесно связан с существованием гетероклинических и гомоклинических решений (см., например, $[8,9]$ ).

Начиная исследование с функционально-дифференциальных включений с выпуклозначной правой частью, удовлетворяющих верхним условиям Каратеодори и условию подлинейного роста, мы распространяем полученные результаты на более широкий класс функционально-дифференциальных включений, правая часть которых является нормальным мультиотображением. Следует отметить, что класс нормальных мультиотображений включает в себя, например, мультиотображения, не обладающие свойством выпуклости значений и удовлетворяющие условию почти полунепрерывности снизу. Еще одним примером рассматриваемого класса являются невыпуклозначные мультиотображения, удовлетворяющие условиям Каратеодори.

В последнем разделе настоящей работы предлагаемый метод решения задачи об асимптотическом поведении траекторий функционально-дифференциальных включений обобщается на случай негладких интегральных направляющих потенциалов.

2. Предварительные сведения. Напомним основные понятия теории многозначных отображений (см., например, $[1,7,13,14])$.

Пусть $\left(X, d_{X}\right)$ и $\left(Y, d_{Y}\right)$ - метрические пространства. Символом $K(Y)$ обозначим множество всех непустых компактных подмножеств пространства $Y$. Если $Y$ - нормированное пространство, то символом $K v(Y)$ обозначается множество всех непустых выпуклых компактных подмножеств $Y$.

Определение 1. Многозначное отображение (мультиотображение) $F: X \rightarrow K(Y)$ называется полунепрерывным сверху (nн. св.) в точке $x_{0} \in X$ если для каждого $V \subset Y$ такого, что $F\left(x_{0}\right) \subset V$ существует $\delta>0$ такое, что из того, что $d_{X}\left(x_{0}, x\right)<\delta$, следует, что $F(x) \subset V$.

Мультиотображение $F: X \rightarrow K(Y)$ называется полунепрерывным сверху (пн. св.), если оно пн. св. в каждой точке $x \in X$.

Определение 2. Мультиотображение $F: X \rightarrow P(Y)$ называется полунепрерывным снизу (nн. cн.) в точке $x_{0} \in X$, если для каждого открытого множества $V \subset Y$ такого, что $F\left(x_{0}\right) \cap V \neq \varnothing$ существует $\delta>0$ такое, что из того, что $d_{X}\left(x_{0}, x\right)<\delta$, следует, что $F(x) \cap V \neq \varnothing$.

Мультиотображение $F: X \rightarrow P(Y)$ называется $n$. сн., если оно пн. сн. в каждой точке $x \in X$.

Определение 3. Мультиотображение $F: X \rightarrow P(Y)$ называется непрерывным, если оно пн. св. и пн. сн.

Пусть $I$ - замкнутое подмножество $\mathbb{R}$, снабженное мерой Лебега $\mu$.

Определение 4. Мультиотображение $F: I \times X \rightarrow K(Y)$ называется почти nн. сн., если существует последовательность компактных непересекающихся множеств $\left\{I_{n}\right\}, I_{n} \subseteq I$, таких, что

(i) $\mu\left(I \backslash \bigcup_{n} I_{n}\right)=0$;

(ii) сужение $F$ на каждое множество $J_{n}=I_{n} \times Y$ является пн. сн. мультиотображением.

Мультиотображение называется мультифункиией, если оно определено на подмножестве $\mathbb{R}$.

Определение 5. Мультифункция $F: I \rightarrow K(Y)$ называется измеримой, если для каждого открытого множества $W \subset Y$ его образ

$$
F^{-1}(W)=\{t \in I: F(t) \subset W\}
$$

является измеримым подмножеством $I$.

Замечание 1. Пн. св. мультифункция является измеримой. Каждая измеримая мультифункция $F: I \rightarrow K(Y)$ имеет измеримое сечение, т. е., существует измеримая функция $f: I \rightarrow Y$ такая, что $f(t) \in F(t)$ для п. в. $t \in I$.

Для каждого $h>0$ обозначим символом $\mathcal{C}$ пространство $C\left([-h, 0] ; \mathbb{R}^{n}\right)$ всех непрерывных функций $x:[-h, 0] \rightarrow \mathbb{R}^{n}$ с обычной нормой. Для данной функции $\psi \in \mathcal{C}$ символом $\mathcal{D}_{\psi}$ будем обозначать множество всех непрерывных функций $x:[-h,+\infty) \rightarrow \mathbb{R}^{n}$ таких, что $x(t)=\psi(t), t \in[-h, 0]$, и сужение $x$ на $\mathbb{R}_{+}=[0,+\infty)$ - абсолютно непрерывно. 
Для функций $x \in \mathcal{D}_{\psi}$ и $t \geqslant 0$ мы будем использовать обозначение $x_{t}$ для функций $x_{t} \in \mathcal{C}$, заданных как $x_{t}(\theta)=x(t+\theta), \theta \in[-h, 0]$.

Определение 6. Говорят, что мультиотображение $F: \mathbb{R}_{+} \times \mathcal{C} \rightarrow K\left(\mathbb{R}^{n}\right)$ удовлетворяет верхним (нижним) условиям Каратеодори, если

$\left(F_{1_{\infty}}\right)$ для каждого $\varphi \in \mathcal{C}$ мультифункция $F(\cdot, \varphi): \mathbb{R}_{+} \rightarrow K\left(\mathbb{R}^{n}\right)$ обладает измеримым сечением; $\left(F_{2_{\infty}}\right)$ для п. в. $t \in \mathbb{R}_{+}$мультиотображение $F(t, \cdot): \mathcal{C} \rightarrow K\left(\mathbb{R}^{n}\right)$ пн. св. (пн. сн.).

Определение 7. Говорят, что мультиотображение $F: \mathbb{R}_{+} \times \mathcal{C} \rightarrow K\left(\mathbb{R}^{n}\right)$ удовлетворяет условиям Каратеодори, если оно удовлетворяет и верхним, и нижним условиям Каратеодори.

Замечание 2. Для того, чтобы выполнялось условие $\left(F_{1_{\infty}}\right)$, достаточно, чтобы мультифункция $F(\cdot, \varphi)$ была измерима для каждого $\varphi \in \mathcal{C}($ см. Замечание 1$)$.

Определение 8. Говорят, что мультиотображение $F: \mathbb{R}_{+} \times \mathcal{C} \rightarrow K\left(\mathbb{R}^{n}\right)$ удовлетворяет условию подлинейного роста, если

$\left(F_{3_{\infty}}\right)$ существует положительная и суммируемая на каждом компактном интервале функция $\alpha(\cdot) \in L_{l o c}^{1}\left(\mathbb{R}_{+}\right)$такая, что для каждого $\varphi \in \mathcal{C}$ имеем

$$
\|F(t, \varphi)\|:=\max _{y \in F(t, \varphi)}\|y\| \leqslant \alpha(t)(1+\|\varphi\|)
$$

для п. в. $t \in \mathbb{R}_{+}$.

Замечание 3. Если мультиотображение $F: \mathbb{R}_{+} \times \mathcal{C} \rightarrow K\left(\mathbb{R}^{n}\right)$ удовлетворяет верхним условиям Каратеодори, то для каждой функции $x \in \mathcal{D}_{\psi}$ мультифункция $t \multimap F\left(t, x_{t}\right), t \in \mathbb{R}_{+}$, обладает локально суммируемым сечением (см., например, $[1,13,14])$.

\section{3. Основные результаты.}

3.1. Выпуклозначный случай. Вначале рассмотрим задачу Коши для функционально-дифференциального включения вида:

$$
\begin{aligned}
x^{\prime}(t) & \in F\left(t, x_{t}\right), \quad \text { п. в. } t \in \mathbb{R}_{+}, \\
x(t) & =\psi(t), \quad t \in[-h, 0],
\end{aligned}
$$

где $\psi \in \mathcal{C}$ является начальной функцией, $F: \mathbb{R}_{+} \times \mathcal{C} \rightarrow K v\left(\mathbb{R}^{n}\right)$ удовлетворяет верхним условиям Каратеодори и условию подлинейного роста. Решением задачи (1), (2) будем называть функцию $x \in \mathcal{D}_{\psi}$, удовлетворяющую включению (1) для п. в. $t \in \mathbb{R}_{+}$.

Заметим, что из основных свойств дифференциальных включений (см., например, $[1,13,14]$ ), следует, что все решения задачи (1), (2) продолжимы на $\mathbb{R}_{+}$и множество этих решений не пусто.

Мы будем исследовать задачу существования решений, удовлетворяющих следующей оценке

$$
\|x(t)\| \leqslant \frac{k}{g(t)}, \quad t \in \mathbb{R}_{+},
$$

где $k>0$ и $g$ - заданная функция.

Обозначим символом $\mathfrak{V}$ совокупность всех непрерывно дифференцируемых функций $V: \mathbb{R}^{n} \rightarrow$ $\mathbb{R}$, удовлетворяющих условию коэриитивности

$$
\lim _{\|x\| \rightarrow+\infty} V(x)=-\infty .
$$

Заметим, что для данной функции $V \in \mathfrak{V}$, для каждого $r>0$ существует $k(r)>r$ такой, что если

$$
\alpha_{r}:=\inf \{V(x),\|x\| \leqslant r\}
$$

то

$$
V(x)<\alpha_{r}, \quad\|x\| \geqslant k(r) .
$$

Теперь пусть $g: \mathbb{R}_{+} \rightarrow \mathbb{R}_{+}$- данная $C^{1}$-функция такая, что

$$
\inf \{g(t), t \in \mathbb{R}\} \geqslant 1 \text {. }
$$


Определение 9. Функция $V \in \mathfrak{V}$ называется интегральным направляюющим потенииалом для включения (1) вдоль функции $g$, если существует

$$
r_{V}>g(0)\|\psi(0)\|
$$

такое, что для каждой функции $x \in \mathcal{D}_{\psi}$, удовлетворяющей условиям

(i) существует наибольшее конечное число $\tau_{1}^{x}>0$ такое, что

$$
g(t)\|x(t)\| \leqslant r_{V} \text { для всех } t \in\left[0, \tau_{1}^{x}\right) ;
$$

(ii) существует наименьшее конечное число $\tau_{*}^{x}>\tau_{1}^{x}$ такое, что

$$
g\left(\tau_{*}^{x}\right)\left\|x\left(\tau_{*}^{x}\right)\right\|=k_{V}:=k\left(r_{V}\right),
$$

(iii) $\left\|x^{\prime}(t)\right\| \leqslant\left\|F\left(t, x_{t}\right)\right\| \quad$ п. в. $t \in \mathbb{R}_{+}$, имеем

$$
\int_{\tau_{\sharp}^{x}}^{\tau_{*}^{x}}\left\langle\nabla V(g(s) x(s)), g^{\prime}(s) x(s)+g(s) f(s)\right\rangle d s \geqslant 0
$$

для каждого локально суммируемого сечения $f(s) \in F\left(s, x_{s}\right)$, где

$$
\tau_{\sharp}^{x}:=\sup \left\{\tau \in\left[\tau_{1}^{x}, \tau_{*}^{x}\right),\|g(\tau) x(\tau)\|=r_{V}\right\} .
$$

Теорема 1. Если $V \in \mathfrak{V}$ является интегральным направляющим потенциалом для включения (1) вдоль функции g, то каждое решение задачи Коши (1), (2) удовлетворяет оценке

$$
\|x(t)\| \leqslant k_{V} \cdot \frac{1}{g(t)}, \quad t \in \mathbb{R}_{+} .
$$

Доказательство. Пусть $x(\cdot)$ - некоторое решение задачи $(1),(2)$ на $\mathbb{R}_{+}$.

Из (7) следует, что существует наибольшее $\tau_{1}^{x}>0$ такое, что

$$
g(t)\|x(t)\| \leqslant r_{V}<k_{V}, \quad t \in\left[0, \tau_{1}^{x}\right) .
$$

Если $\tau_{1}^{x}=+\infty$, то

и утверждение верно.

$$
\|x(t)\|<k_{V} \cdot \frac{1}{g(t)}, \quad t \in \mathbb{R}_{+}
$$

Если $t_{1}^{x}<+\infty$, то оценка (10) справедлива только на ограниченном интервале. Покажем, что

$$
g(t)\|x(t)\| \leqslant k_{V}, \quad t \in \mathbb{R}_{+} .
$$

Предположим противное, что найдется $\tau_{2}^{x}>\tau_{1}^{x}$ такое, что

$$
g\left(\tau_{2}^{x}\right)\left\|x\left(\tau_{2}^{x}\right)\right\|>k_{V}
$$

Из (10) и (13) следует, что существует наименьшее $\tau_{1}^{x}<\tau_{*}^{x}<\tau_{2}^{x}$, для которого

$$
g\left(\tau_{*}^{x}\right)\left\|x\left(\tau_{*}^{x}\right)\right\|=k_{V} .
$$

Возьмем

$$
\tau_{\sharp}^{x}=\sup \left\{\tau \in\left[\tau_{1}^{x}, \tau_{*}^{x}\right), g(\tau)\|x(\tau)\|=r_{V}\right\},
$$

имеем

Из (5) получаем оценку

$$
g\left(\tau_{\sharp}^{x}\right)\left\|x\left(\tau_{\sharp}^{x}\right)\right\|=r_{V} .
$$

Из определения 9 вытекает, что

$$
V\left(g\left(\tau_{\sharp}^{x}\right) x\left(\tau_{\sharp}^{x}\right)\right) \geqslant \alpha_{r_{V}} .
$$

$$
V\left(g\left(\tau_{*}^{x}\right) x\left(\tau_{*}^{x}\right)\right)-V\left(g\left(\tau_{\sharp}^{x}\right) x\left(\tau_{\sharp}^{x}\right)\right)=\int_{\tau_{\sharp}^{x}}^{\tau_{*}^{x}}\left\langle\nabla V(g(s) x(s)), g^{\prime}(s) x(s)+g(s) x^{\prime}(s)\right\rangle d s \geqslant 0 .
$$


С учетом соотношений (6), (14) и (15), имеем

$$
\alpha_{r_{V}}>V\left(g\left(\tau_{*}^{x}\right) x\left(\tau_{*}^{x}\right)\right) \geqslant V\left(g\left(\tau_{\sharp}^{x}\right) x\left(\tau_{\sharp}^{x}\right)\right) \geqslant \alpha_{r_{V}} .
$$

Полученное противоречие доказывает справедливость оценки (12).

3.2. Невыпуклозначный случай. Теперь мы будем рассматривать нашу задачу для класса функционально-дифференциальных включений с невыпуклозначными правыми частями. Определим основные понятия (см. [13]).

Определение 10. Ограниченное мультиотображение $R: \mathbb{R}_{+} \times \mathcal{C} \rightarrow K\left(\mathbb{R}^{n}\right)$ называется нормальным, если существует мультиотображение $F: \mathbb{R}_{+} \times \mathcal{C} \rightarrow K v\left(\mathbb{R}^{n}\right)$, называемое нормальным квазисечением мультиотображения $R$, удовлетворяющее следующим условиям:

(i) мультиотображение $F$ удовлетворяет верхним условиям Каратеодори и условию подлинейного роста;

(ii) $F(t, \varphi) \cap R(t, \varphi) \neq \varnothing$ для всех $t \in I, \varphi \in \mathcal{C}$;

(iii) каждое решение $x \in \mathcal{D}_{\psi}$ задачи $(1),(2)$ с правой частью $F$ является решением задачи

$$
\begin{gathered}
x^{\prime}(t) \in R\left(t, x_{t}\right) \quad \text { п. в. } t \in \mathbb{R}_{+}, \\
x(t)=\psi(t), \quad t \in[-h, 0] .
\end{gathered}
$$

Замечание 4. (см., например, $[11,13])$ Очевидно, что каждое ограниченное мультиотображение $F: \mathbb{R}_{+} \times \mathcal{C} \rightarrow K v\left(\mathbb{R}^{n}\right)$, удовлетворяющее верхним условиям Каратеодори, является нормальным мультиотображением. Каждое ограниченное почти пн. сн. мультиотображение $R: \mathbb{R}_{+} \times \mathcal{C} \rightarrow$ $K\left(\mathbb{R}^{n}\right)$ является нормальным мультиотображением. Каждое ограниченное мультиотображение $R: \mathbb{R}_{+} \times \mathcal{C} \rightarrow K\left(\mathbb{R}^{n}\right)$, удовлетворяющее условиям Каратеодори, является нормальным мультиотображением.

Теорема 2. Если $V \in \mathfrak{V}$ является интегральным направляющим потенииалом для включения (16) вдоль функиии g, то существует по крайней мере одно решение задачи Коши (16), (17), удовлетворяющее оченке (9).

Доказательство. Пусть $F$ является нормальным квазисечением мультиотображения $R$.

Рассмотрим решение $x: \mathbb{R}^{+} \rightarrow \mathbb{R}^{n}$ задачи Коши

$$
\begin{gathered}
x^{\prime}(t) \in F\left(t, x_{t}\right) \quad \text { п. в. } t \in \mathbb{R}_{+}, \\
x(t)=\psi(t), \quad t \in[-h, 0] .
\end{gathered}
$$

Согласно условию (iii) определения 10 оно является и решением задачи Коши (16), (17). Если для него $\tau_{1}^{x}=+\infty$, то верна оценка (9).

Если $\tau_{1}^{x}<+\infty$, то

$$
\int_{\tau_{\sharp}^{x}}^{\tau_{*}^{x}}\left\langle\nabla V(g(s) x(s)), g^{\prime}(s) x(s)+g(s) x^{\prime}(s)\right\rangle d s \geqslant 0,
$$

так как $x^{\prime}(s) \in R\left(s, x_{s}\right)$ является локально суммируемым сечением.

Тогда, повторяя рассуждения, используемые при доказательстве теоремы 1 , мы получим искомую оценку для данного решения $x$ задачи Коши (16), (17).

В силу замечания 4 справедливы следующие утверждения.

Теорема 3. Предположим, что правая часть функционально-дифференииального включения (16) является ограниченным почти пн. сн. мультиотображением $R: \mathbb{R}_{+} \times \mathcal{C} \rightarrow K\left(\mathbb{R}^{n}\right)$. Если $V \in \mathfrak{V}$ является интегральным направляющим потенииалом для включения (16) вдоль функиии g, то существует по крайней мере одно решение соответствующей задачи Коши, удовлетворлющее оценке (9). 
Теорема 4. Предположим, что правая часть функиионально-дифференциального включения (16) является ограниченным мультиотображсением $R: \mathbb{R}_{+} \times \mathcal{C} \rightarrow K\left(\mathbb{R}^{n}\right)$, удовлетворяющим условиям Каратеодори. Если $V \in \mathfrak{V}$ является интегральным направляющим потенциалом для включения (16) вдоль функиии g, то существует по крайней мере одно решение соответствующей задачи Коши, удовлетворяющее оченке (9).

3.3. Негладкий случай. Приведенные выше результаты могут быть распространены и на случай негладких направляющих потенциалов.

Напомним некоторые понятия негладкого анализа (см. [12]).

Пусть $V$ является локально липшицевой функцией в пространстве $\mathbb{R}^{n}$. Для $x_{0} \in \mathbb{R}^{n}$ и $\nu \in \mathbb{R}^{n}$ обобщенная производная Кларка $V^{0}\left(x_{0} ; \nu\right)$ в точке $x_{0}$ по направлению $\nu$ задается формулой

$$
V^{0}\left(x_{0} ; \nu\right)=\varlimsup_{\substack{x \rightarrow x_{0} \\ t \rightarrow 0+}} \frac{V(x+t \nu)-V(x)}{t}
$$

где $x \in \mathbb{R}^{n}$. Тогда обобщенный градиент Кларка $\partial V(x)$ функции $V$ в точке $x_{0}$ определяется следующим образом:

$$
\partial V\left(x_{0}\right)=\left\{x \in \mathbb{R}^{n}:(x, \nu) \leqslant V^{0}\left(x_{0} ; \nu\right) \text { для всех } \nu \in \mathbb{R}^{n}\right\} .
$$

Известно, что мультиотображение $\partial V(x)$ является пн. св. и имеет выпуклые компактные значения. Следовательно, для каждой непрерывной функции $x: \mathbb{R} \rightarrow \mathbb{R}^{n}$ мультифункция $t \in \mathbb{R} \rightarrow$ $\partial V(x(t))$ имеет локально суммируемое сечение.

Напомним также (см., например, [12]), что локально липшицева функция $V: \mathbb{R}^{n} \rightarrow \mathbb{R}$ называется регулярной, если для каждого $x \in \mathbb{R}^{n}$ и $\nu \in \mathbb{R}^{n}$ существует производная $V^{\prime}(x, \nu)$, и она совпадает с $V^{0}(x, \nu)$. В частности, известно, что выпуклые функции являются регулярными.

Обозначим символом $\mathcal{V}$ совокупность всех регулярных функций $V: \mathbb{R}^{n} \rightarrow \mathbb{R}$, удовлетворяющих условию коэрцитивности (4).

Как и ранее, пусть дана функция $V \in \mathcal{V}$, такая, что для каждого $r>0$ существует $k(r)>r$ такое, что выполняется соотношение (6).

Пусть $g: \mathbb{R}_{+} \rightarrow \mathbb{R}_{+}$- заданная $C^{1}$-функция такая, что

$$
\inf \{g(t), t \in \mathbb{R}\} \geqslant 1 .
$$

Определение 11. Функция $V \in \mathcal{V}$ называется негладким интегральным направляющим потенциалом для включения (16) вдоль функции $g$, если существует

$$
r_{V}>g(0)\|\psi(0)\|
$$

такое, что для каждой функции $x \in \mathcal{D}_{\psi}$, удовлетворяющей условиям

(i) существует наибольшее конечное число $\tau_{1}^{x}>0$ такое, что

$$
g(t)\|x(t)\| \leqslant r_{V} \text { для всех } t \in\left[0, \tau_{1}^{x}\right) ;
$$

(ii) существует наименьшее конечное число $\tau_{*}^{x}>\tau_{1}^{x}$ такое, что

$$
g\left(\tau_{*}^{x}\right)\left\|x\left(\tau_{*}^{x}\right)\right\|=k_{V}:=k\left(r_{V}\right),
$$

(iii) $\left\|x^{\prime}(t)\right\| \leqslant\left\|R\left(t, x_{t}\right)\right\|$ п. в. $t \in \mathbb{R}_{+}$,

имеем

$$
\int_{\tau_{\sharp}^{x}}^{\tau_{*}^{x}}\left\langle v(s), g^{\prime}(s) x(s)+g(s) f(s)\right\rangle d s \geqslant 0
$$

для каждого локально суммируемого сечения $v(s) \in \partial V(g(s) x(s))$ и $f(s) \in R\left(s, x_{s}\right)$, где

$$
\tau_{\sharp}^{x}:=\sup \left\{\tau \in\left[\tau_{1}^{x}, \tau_{*}^{x}\right),\|g(\tau) x(\tau)\|=r_{V}\right\} .
$$

Применяя аналогичные рассуждения, что и при доказательстве теорем 1 и 2 , легко получить следующий результат. 
Теорема 5. Если $V \in \mathcal{V}$ является негладким интегральным направляющим потенииалом для включения (16) вдоль функиии $g$, то существует по крайней мере одно решение задачи Коши (16), (17), удовлетворяющее оченке (9).

Замечание 5. Утверждение справедливо и для случая, когда правая часть $R: \mathbb{R}_{+} \times \mathcal{C} \rightarrow$ $K\left(\mathbb{R}^{n}\right)$ функционально-дифференциального включения (16) является ограниченным почти пн. сн. мультиотображением или ограниченным мультиотображением, удовлетворяющим условиям Каратеодори.

\section{СПИСОК ЛИТЕРАТУРЫ}

1. Борисович Ю. Г., Гельман Б. Д., Мышкис А. Д., Обуховский В. В. Введение в теорию многозначных отображений и дифференциальных включений. - М.: Либроком, 2011.

2. Корнев С. В. Асимптотическое поведение решений дифференциальных включений с невыпуклой правой частью// Вестн. Воронеж. гос. ун-та. Сер. Физ. Мат. - 2016. - 1. - С. 96-104.

3. Корнев C. В., Лой H. В. Метод многолистных направляющих функций в задаче о бифуркации решений дифференциальных уравнений// Вестн. Тамбов. ун-та. Сер. Естеств. техн. науки. - 2016. - 21, № 2. - C. 390-401.

4. Корнев С. В., Обуховский В. В. Асимптотическое поведение решений дифференциальных включений и метод направляющих функций// Диффер. уравн. - 2015. - 51, № 6. - С. 700-705.

5. Красносельский М. А. Оператор сдвига по траекториям дифференциальных уравнений. - М.: Наука, 1966.

6. Красносельский М. А., Забрейко П. П. Геометрические методы нелинейного анализа. - М.: Наука, 1975.

7. Arutyunov A. V., Obukhovskii V. Convex and Set-Valued Analysis. - Berlin-Boston: De Gruyter, 2017.

8. Avramescu C. Existence problems for homoclinic solutions// Abstr. Appl. Anal. - 2002. - 7. - P. 1-29.

9. Avramescu C. Evanescent solutions of linear ordinary differential equations// Electr. J. Qualit. Theory Differ. Equ. - 2002. - 9. - P. 1-12.

10. Avramescu C. Asymptotic behavior of solutions of nonlinear differential equations and generalized guiding functions// Electr. J. Qualit. Theory Differ. Equ. — 2003. - 13. — P. 1-9.

11. Bressan A., Colombo G. Selections and representations of multifunctions in paracompact spaces// Stud. Math. - 1992. - 102. - P. 209-216.

12. Clarke F. H. Optimization and Nonsmooth Analysis. - Philadelphia: SIAM, 1990.

13. Górniewicz L. Topological Fixed Point Theory of Multivalued Mappings. - Berlin: Springer, 2006.

14. Kamenskii M., Obukhovskii V., Zecca P. Condensing Multivalued Maps and Semilinear Differential Inclusions in Banach Spaces. - Berlin-New York: de Gruyter, 2001.

15. Kornev S. V., Liou Y. C. Multivalent guiding functions in the bifurcation problem of differential inclusions// J. Nonlin. Sci. Appl. — 2016. - 9. - P. 5259-5270.

16. Kornev S. V., Loi N. V., Obukhovskii V. V., Wen C. F. Random nonsmooth integral guiding functions and asymptotic behavior of trajectories for random differential inclusions// J. Nonlin. Convex Anal. - 2018. - 19, № 3. - P. 493-500.

17. Kornev S. V., Obukhovskii V. V. Asymptotic behavior of solutions for inclusions with causal multioperators and the method of integral guiding potentials// Вестн. Тамбов. ун-та. Сер. Естеств. техн. науки. - 2018. -23 , № 122 . - C. $136-144$.

18. Kornev S., Obukhovskii V., Yao J. C. On asymptotics of solutions for a class of functional differential inclusions// Discuss. Math. - 2014. - 34. - P. 219-227.

19. Kryszewski W. Homotopy Properties of Set-Valued Mappings. - Torun: Univ. N. Copernicus Publishing, 1997.

20. Loi N. V., Obukhovskii V., Yao J. C. A bifurcation of solutions of nonlinear Fredholm inclusions involving $C J$-multimaps with applications to feedback control systems// Set-Valued Var. Anal. — 2013. — 21. — P. 247-269.

21. Loi N. V., Obukhovskii V., Yao J. C. A multiparameter global bifurcation theorem with application to a feedback control system// Fixed Point Theory. — 2015. - 16. - P. 353-370.

22. Obukhovskii V., Kamenskii M., Kornev S., Liou Y. C. On asymptotics of solutions for a class of differential inclusions with a regular right-hand part// J. Nonlin. Convex Anal. — 2017. — 18, № 5. — P. 967-975. 
23. Obukhovskii V., Loi N. V., Kornev S. Existence and global bifurcation of solutions for a class of operatordifferential inclusions// Differ. Equ. Dynam. Syst. — 2012. — 20, № 3. - P. 285-300.

24. Obukhovskii V., Zecca P., Loi N. V., Kornev S. Method of Guiding Functions in Problems of Nonlinear Analysis. - Berlin: Springer-Verlag, 2013.

Безмельницына Юлия Евгеньевна

Воронежский государственный педагогический университет

E-mail: bezmelnicyna@inbox.ru

Корнев Сергей Викторович

Воронежский государственный педагогический университет

E-mail: kornev_vrn@rambler.ru

Обуховский Валерий Владимирович

Воронежский государственный педагогический университет

E-mail: valerio-ob2000@mail.ru 